\title{
Review: home visiting programmes that include $\geqslant 1$ postnatal home visit are associated with improved quality of home environment and parenting
}

Kendrick D, Elkan R, Hewitt M, et al. Does home visiting improve parenting and the quality of the home environment? A systematic review and meta analysis. Arch Dis Child 2000 Jun;82:443-51.

\section{QUESTION: Do home visiting programmes improve quality of home environment and parenting?}

\section{Data sources}

Studies were identified by searching Medline (1966 to July 1996), CINAHL (1982 to July 1996), EMBASE/ Excerpta Medica (1980 to October 1996), and the Cochrane Library. The journal Health Visitor was handsearched (1982-97), bibliographies of relevant reviews were checked, key individuals and organisations were contacted, and advertisements were placed in relevant journals to identify unpublished studies.

\section{Study selection}

Randomised controlled trials or quasi-experimental studies that had a control group were included if they evaluated a home visiting programme, the programme included $\geqslant 1$ postnatal home visit, the tasks undertaken in the home visit were within the practice of British health visitors (eg, social support and facilitation of mother-child interaction), and outcomes relevant to British home visiting were reported (eg, quality of home environment and parent-child interaction).

\section{Data extraction}

Data were extracted on study purpose, design, sample size determination, description of participants, randomisation and stratification, use of a comparison group, management procedures, blinding, loss to follow up, and evaluation. 3 reviewers blinded to study authors, results, and conclusions assessed the quality of individual studies using the Reisch scale (score of $0-1$, with higher scores representing higher quality).

\section{Main results}

34 studies met the inclusion criteria: 17 reported Home Observation for Measurement of the Environment (HOME) scores, 27 reported other parenting outcomes, and 10 reported both types of outcomes. 12 of 17 studies reporting HOME scores provided enough data to be included in a meta-analysis, which used Fisher's method based on reported $\mathrm{p}$ values. Home visiting was associated with improved HOME scores $(p<0.001)$; results were similar when the analyses were restricted to randomised studies $(\mathrm{p}<0.001)$ and higher quality studies (Reisch scores $\geqslant 0.5)(\mathrm{p}<0.001)$.

21 of the 27 studies that reported other measures of parenting found improvements associated with home visiting. 12 of 17 studies found improved mother-child interactions with home visiting (ie, increased involvement and reciprocal interaction, responsiveness to child's behaviour, quantity and type of interaction, conversation, positive feedback and praise for the child, and a more positive attitude towards the child; and decreased rates of reported difficulties in the relationship and negative inter- actions). 5 studies found no group differences for motherchild attachment, maternal interaction, parental warmth, verbal praise, and shared activities.

Home visiting was associated with more positive or realistic developmental expectations of the child (4 of 5 studies); more maternal involvement in schooling and more stimulation to promote future success (2 of 2 studies); and parental stimulation of the child using books, games, or toys (3 of 5 studies).

\section{Conclusion}

Home visiting programmes that include $\geqslant 1$ postnatal visit are associated with improved quality of the home environment and improved parenting.
Source of funding: UK National Health Service $R \mathcal{E}$ D D Health Technology Assessment Programme.

For correspondence: Dr D Kendrick, Division of General Practice, University of Nottingham, Queen's Medical Centre,

Nottingham NG7 $2 \mathrm{UH}$, UK. Fax $+44(0) 115$ 9709389 .

A modified version of this abstract appears in Evidence-Based Mental Health

\section{COMMENTARY}

The results of this systematic review by Kendrick $e t$ al are consistent with previous reviews that show the benefits of home visiting programmes. ${ }^{1-3}$ Most of the studies included in this review focused on US families from disadvantaged backgrounds; however, studies from the UK, Canada, and Scandinavia had consistent results. Nevertheless, the findings should not be generalised without attention to specific settings and populations. The quality of the 17 studies that used the HOME outcome measure varied from poor to good (Reisch scores of 0.18 to 0.79 out of a possible score of 1 , with a mean score of 0.46). A common weakness in methodology was unblinded outcome assessment, which may have introduced bias. However, the similar results obtained when lower quality studies were excluded, reinforce the strength of the findings. Because the studies that used other parenting outcomes did not present findings in a way that permitted meta-analysis, the authors simply reported the number of studies that found improvements. It would have been helpful if the authors had commented on the quality of these studies as well. It could be that the better quality studies were those that found no improvements with home visiting.

The findings are relevant to health visitors, community and public health nurses, midwives, neonatal nurses, social workers, and anyone involved in working with young children and their families. The study is also of interest to organisations and agencies involved in the planning and implementation of early intervention programmes aimed at improving parenting.

Eight studies used lay workers and the results of these studies were similar to those that used professional visitors; however, because several of these studies had poor quality ( 5 had scores $<0.50$ ), the use of lay workers requires further study. It is also interesting that studies with follow up periods $<2$ years had more favourable results than those with follow up periods $\geqslant 2$ years. This may suggest that the effect of intervention programmes diminishes over time. More comprehensive programmes supporting families over a longer period of time may be worth considering.

Pauline Raynor, RN, RMW, RHV, BA(Hons) Lecturer, Centre for Evidence Based Nursing University of York, York, UK

1 Hodnett ED, Roberts I. Home-based social support for socially disadvantaged mothers. Cochrane Database Syst Rev 2000;(2):CD000107 (latest version 26 Oct 1998).

2 Roberts I, Kramer MS, Suissa S. Does home visiting prevent childhood injury? A systematic review of randomised controlled trials. BMJ 1996;312:29-33.

3 Ciliska D, Hayward S, Thomas H, et al. A systematic overview of the effectiveness of home visiting as a delivery strategy for public health nursing interventions. Can J Public Health $1996 ; 87: 193-8$. 